\title{
Current management and prognostic features for gastrointestinal stromal tumor (GIST)
}

\author{
Gurpreet Lamba, Ridhi Gupta, Byung Lee, Samir Ambrale and Delong Liu*
}

\begin{abstract}
Stromal or mesenchymal neoplasms affecting the gastrointestinal (Gl) tract have undergone a remarkable evolution in how they are perceived, classified, approached, diagnosed and managed over the last 30 years. Gastrointestinal stromal tumors (GIST) account for approximately $1 \%$ to $3 \%$ of all malignant GI tumors. The clinical features can vary depending on the anatomic location, size and aggressiveness of the tumor. Metastatic GIST represents a successful example of molecular targeted therapy. In this comprehensive review, we discuss the epidemiology, clinical features and diagnostic modalities for GIST. We also describe treatment options for early stage, locally advanced and metastatic GIST. Indications for neoadjuvant and adjuvant therapy along with duration of therapy are also explained. A brief discussion of latest biomarkers and updates from recent meetings is also provided.
\end{abstract}

\section{Introduction}

Stromal or mesenchymal neoplasms affecting the GI tract have undergone a remarkable evolution in how they are perceived, classified, approached, diagnosed and managed over the last 30 years. A major breakthrough occurred with the discovery of expression of the CD117 antigen by almost all gastrointestinal stromal tumors (GIST)[1]. The other group of spindle cell neoplasms arising in the GI tract includes lipomas, schwannomas, hemangiomas, usual leiomyomas and leiomyosarcomas are typically CD117-negative [2]. The CD117 molecule is part of the KIT (c-kit) receptor tyrosine kinase that is a product of the KIT proto-oncogene (Figure 1). GIST research and clinical care sets another great example of translational research that turns laboratory discovery to successful clinical application. From this fundamental mechanistic understanding of GIST, a series of worldwide investigations and trials have developed novel and effective ways to approach patients with this disease. In this review, we discuss the basics of GIST and highlight recent advances and their relevance to current clinical practice as well as future directions.

\section{Epidemiology}

GISTs account for approximately $1 \%$ to $3 \%$ of all malignant GI tumors [3]. Epidemiologic data such as that from Surveillance, Epidemiology and End Results (SEER) program are difficult to interpret since the earlier definition of "malignant GIST" was derived from criteria published in 1990, before GIST was molecularly characterized [4]. The estimated incidence of GIST has been revised upward to approximately 5,000 new cases per year in the United States (US) [5,6]. The most dependable international epidemiologic data are from population-based studies that reexamined all cases of potential GIST [710]. These studies reported annual incidence of GIST ranging from 11 to 14.5 per million population. More recent studies suggest that the incidentally detected subcentimeter gastric GIST lesions may be more frequent than expected [11,12].

\section{Clinical presentation}

The clinical features can vary depending on the anatomic location, size and aggressiveness of the tumor. Most symptomatic patients have tumors larger than $5 \mathrm{~cm}$ in maximal dimension. In a series of cases with leiomyomas and leiomyosarcoma (without separation of the GISTs), there were three major presentations [13], GI bleeding (40\%), abdominal mass (40\%) and abdominal pain (20\%). Two-thirds of patients had GI bleeding while 25 to $40 \%$ presented with an intestinal obstruction. Intestinal perforation can also occur uncommonly. Rare patients have been described

\footnotetext{
* Correspondence: delong_liu@nymc.edu

Division of Oncology/Hematology, New York Medical College and Westchester Medical Center, Valhalla, NY 10595, USA
} 


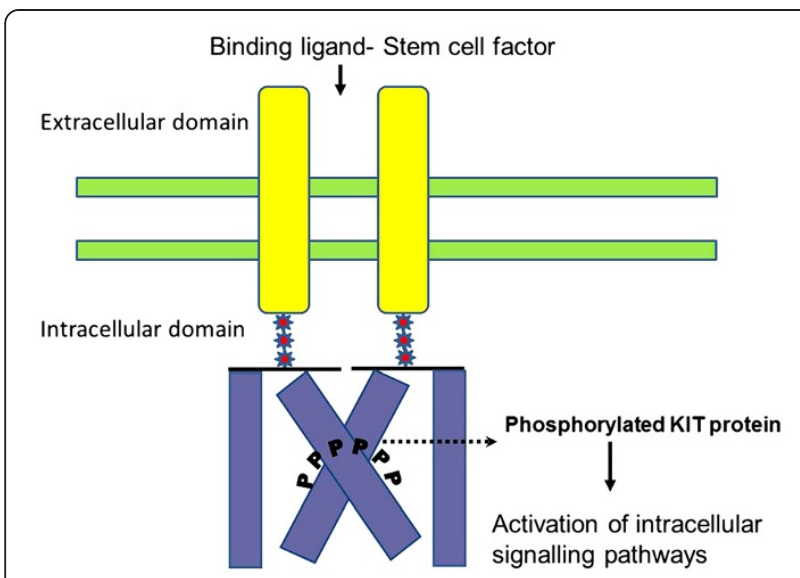

Figure 1 The structure of KIT/CD117 and the signal

transduction. KIT is a transmembrane receptor type tyrosine knase. The stem cell factor/KIT ligand binds to KIT and activates the KIT

tyrosine kinase. The phosphorylated (activated) KIT then activates its substrates which lead to cell proliferation.

who present with severe hypoglycemia due to paraneoplastic production of insulin like growth factor II [14]. Other symptoms at presentation may include nausea, vomiting, anorexia, and weight loss. The vast majority of GIST metastases at presentation are intra-abdominal, either to the liver, omentum or peritoneal cavity [15]. Metastases to lymph nodes or to extra-abdominal sites via lymphatics are rare.

\section{Diagnostic imaging}

GIST should always be among the differential diagnosis of an intra-abdominal non-epithelial malignancy. CT is essential for evaluating the primary tumor and for accurate staging. Magnetic resonance imaging (MRI) has a comparable diagnostic yield [16] and lacks radiation exposure. However, CT is preferred initially for screening and staging. CT is better at global evaluation of the abdomen, especially the hollow viscera, than MRI. MRI may be preferred for GISTs at specific sites, such as rectum or liver. Tumors that are greater than $5 \mathrm{~cm}$, lobulated, enhance heterogeneously, and have mesenteric fat infiltration, ulceration, regional lymphadenopathy, or an exophytic growth pattern on CT are more likely to metastasize [17-21]. In contrast, GISTs with less metastatic potential tend to enhance in a homogeneous pattern, and often show an endoluminal growth pattern. CT or MRI scanning can assess the decrease in lesion density which can be an early marker of beneficial response in GIST patients treated with TKI drugs [22]. Routine clinical practice rarely requires Positron Emission Tomography (PET) imaging of GIST for clinical care [23]. PET imaging may have the advantage of detecting small lesions at least $1 \mathrm{~cm}$ in size because neither the normal bowel nor omentum takes up the fluorodeoxyglucose (FDG) tracer with excess avidity. The reported sensitivity of PET for GIST is 86 to $100 \%$ $[24,25]$. PET can be useful for detecting an unknown primary site or resolving ambiguities from CT [26]. On upper GI endoscopy, a smooth, mucosa-lined protrusion of the bowel wall, with or without signs of bleeding and ulceration may be seen [27]. However, endoscopic biopsies using standard techniques usually do not obtain sufficient tissue for a definite diagnosis [28]. Endoscopic ultrasound (EUS) -guided fine-needle biopsy forceps also may not yield enough tissue, but might exclude other lesions that arise sub-mucosally. Snare biopsies can result in perforation and generally should be avoided except in carefully selected cases [28]. A preoperative biopsy is not generally recommended for a resectable lesion in which there is a high suspicion for GIST, and the patient is otherwise operable. However, a biopsy should be done to confirm the diagnosis particularly when metastatic disease is present or suspected. If preoperative imatinib is considered in a patient who has a large locally advanced lesion thought to represent GIST, a biopsy should be done. An EUS-guided biopsy (in carefully selected patients and preferably of the primary lesions) is more desirable than a percutaneous biopsy [29].

\section{Histopathology and cytology}

Differentiation between GISTs and other tumors is typically based upon immunohistochemistry (IHC) and molecular analysis. Histologic findings seen on hematoxylin and eosin-stained sections do not reliably or specifically relate to the immunophenotype nor the molecular genetics of the lesions [30]. GISTs characteristically stain positive for CD117 antigen (KIT) on IHC assays. The level of expression can vary from generally diffuse and strong (most common in the spindle cell subtype) to focal and weakly positive in a dot-like pattern (the epithelioid subtype) [5]. CD34 expression is not specific for GIST and it can also be seen in desmoid tumors [5,31,32]. About $95 \%$ of GISTs are KIT-positive, while 60 to $70 \%$ are positive for CD34, 30 to $40 \%$ are positive for smooth muscle actin, $5 \%$ for S-100 protein, and 1 to $2 \%$ are positive for desmin or keratin [2,5,32]. Identification of CD117-negative GIST remains a diagnostic challenge, and these are most likely to be driven by alternative kinases like PDGFRA [33]. The antigen known as DOG1 ("discovered on GIST-1") can also help to identify certain KIT-negative GIST lesions as DOG-1 expression is quite specific for GIST $[34,35]$.

\section{Prognostic features of GIST}

The current consensus is to treat all GISTs, including those with a benign appearance by conventional histopathologic criteria, as having the potential to behave in a malignant fashion [5,23]. This is due to the biologic 
behavior of GIST, which can be highly variable and with long follow-up, virtually all GISTs have the potential for malignant behavior, even those $2 \mathrm{~cm}$ or less with bland histologic features [2]. Thus, it is not appropriate to define any GIST as "benign" per se [5,36,37]. The most reliable prognostic factors for GIST are size of the primary tumor and the mitotic index. Additionally, recurrence and survival rates can be affected by the location of the primary GIST lesion (e.g. with small bowel and rectal primary GIST demonstrate worse prognosis than gastric GISTs) [36,37]. PDGFRA mutations (almost always in gastric primaries) appear to be a very favorable prognostic factor for low risk of recurrence [38]. Among patients with GIST, histologic type may also impact prognosis. In a report of 48 patients, the five-year recurrence-free survival rate was significantly higher among patients with spindle cell as compared to epithelioid or mixed histology [39]. However, others report a prognostic influence of the degree of cellularity but not histologic subtype [40,41].

\section{Management paradigms for early-stage GIST}

The natural history of early-stage GIST has been documented in single-institution studies. Two hundred patients with GIST were followed prospectively at the Memorial Sloan-Kettering Cancer Center [15]. Eighty of these patients who had primary disease were managed with complete surgical resection. This group of patients demonstrated a 5-year disease-specific survival rate of $54 \%$. On multivariate analysis, large tumor size $(>10 \mathrm{~cm})$ was the only negative predictive factor on disease-specific survival. Definitive surgery remains the treatment of choice for patients with localized GIST. Complete surgical resection is recommended for small gastric GISTs $<2 \mathrm{~cm}$ at high risk of recurrence based upon EUS appearance (irregular borders, cystic spaces, ulceration, echogenic foci, or heterogeneity in appearance). For tumors that lack these features, endoscopic surveillance is an option. However, initial therapy with imatinib may be preferred if a tumor is borderline resectable, or if resection would necessitate extensive organ disruption. There is no specific consensus or guideline for the use of neoadjuvant imatinib at this time. However, this could serve as the initial therapeutic intervention, with follow-up at close intervals to ensure appropriate response to therapy. In these situations, early assessment of therapeutic response by ${ }^{18} \mathrm{FDG}$-PET scanning could be very valuable to confirm the response to imatinib. After maximal response (usually occurring within 4 to 6 months), definitive surgery could be performed [42].

\section{Adjuvant therapy for resected early-stage GIST}

A large multicenter landmark phase 3 trial (ACOSOG study Z9001) appears to support the use of imatinib therapy in patients with larger, fully resected, primary localized GIST lesions at significant risk of relapse [43]. Seven hundred and thirteen adults with a completely resected primary GIST $(>=3 \mathrm{~cm}, \mathrm{KIT}+$ ) were randomly assigned to one year of adjuvant imatinib (400 mg daily) or placebo [43]. The trial was stopped at an early, preplanned, event-based interim analysis because of the positive outcome. At a median follow-up of 20 months, 30 patients in the imatinib group recurred or died, versus 70 in the placebo group (8 versus $20 \%$ ). The oneyear relapse-free survival (RFS) rate was 98 versus $83 \%$ favoring imatinib, with a hazard ratio for RFS of 0.35 (95\% CI 0.22 to 0.53 ). In a later analysis, the benefit was greatest in those with high-risk disease (relapse rate 47 versus $19 \%$ for placebo and imatinib, respectively); for moderate risk disease it was 14 versus $5 \%$, respectively [44]. Despite prolonged RFS, no benefit in overall survival (OS) was noted with short follow-up. Furthermore, after the study was unblinded, all patients randomized to placebo were allowed to crossover to active treatment, thus obscuring any potential differences in overall survival between the groups. Thus, it remains unclear whether imatinib is simply delaying or really preventing relapses. The trial tested only the $400 \mathrm{mg}$ daily dose. Patients with advanced GIST and KIT exon 9 mutations do better with $800 \mathrm{mg}$ daily doses. Whether doses greater than $400 \mathrm{mg}$ should be used in the adjuvant setting will require prospective study. Although they were excluded, these results may also be significant for the $4 \%$ of patients with GISTs that lack KIT overexpression, but have mutations in KIT or PDGFRA, and can respond to imatinib [45]. Contrary to this, patients who lack detectable KIT or PDGFRA mutations or who have specific mutations that are known to be resistant to imatinib may not benefit. Whether such patients should be identified prospectively and specifically excluded from receiving adjuvant imatinib is unclear.

The FDA approved the use of imatinib as adjuvant therapy without any qualification or restriction following resection of primary GIST $\geq 3 \mathrm{~cm}$ in size, but the European Medicines Agency approved adjuvant imatinib only for the subset of GIST patients with primary disease that is judged to be "at significant risk of recurrence" following resection.

\section{Estimation of recurrence risk}

Several criteria have been proposed, originally to classify the malignant potential of a GIST. Tumor size, mitotic rate, and site of tumor origin have gained the greatest acceptance as being predictive of outcome [46]. Risk stratification models have also been proposed to distinguish prognosis in resected GIST [39]. Tumors arising from the small bowel, colon, rectum, or mesentery are associated with less favorable outcomes than those 
arising from the stomach [37]. Thus, each case must be approached individually, balancing the estimated probability of a disease recurrence.

Adjuvant imatinib for 1 year certainly has a major impact on disease control rates, but these differences appear to fade with increased rates of recurrence noted on discontinuation of the imatinib dosing. It is possible that a longer duration of adjuvant therapy might further improve clinical outcomes. A small study of adjuvant therapy for 2 years in Seoul, Korea has shown much higher rates of disease control than in the 1-year study, consistent with biological expectations [47]. The results of a trial by the Scandinavian Sarcoma Group (SSG) XVIII trial were presented at the 2011 American Society of Clinical Oncology (ASCO) meeting and then published in 2012. This trial compared 36 versus 12 months of imatinib (400 mg daily) in 400 patients with high-risk resected GIST [48]. High-risk was defined as having at least one of: tumor size $>10 \mathrm{~cm}$, mitotic count $>10 / 50$ high-power fields (hpf), tumor size $>5 \mathrm{~cm}$ with mitotic rate $>5 / 50 \mathrm{hpf}$, or tumor rupture. About one-half of the enrolled patients had gastric primary tumors. At a median follow-up of 54 months, prolonged treatment was associated with a significant improvement in RFS, the primary endpoint (five-year RFS 66 versus 48\%, HR 0.46, $95 \%$ CI 0.32 to 0.65 ) as well as OS (92 versus $82 \%$, HR $0.45,85 \% \mathrm{CI} 0.22$ to 0.89 ). However, twice as many patients discontinued imatinib for reasons other than disease progression in the prolonged therapy group (26 versus 13\%). This dataset attempts to establish 36 months of adjuvant imatinib as a new standard for patients with high-risk resected GIST. In both groups, within 6 to 12 months of discontinuing adjuvant imatinib, rates of disease recurrence were similarly increased. This and previous findings seem to support the notion that recurrences are just delayed, rather than being prevented. The question now is whether treatment should be continued for longer than three years. One study assessed costeffectiveness of 3 years versus 1 year of adjuvant imatinib in the US from a payer's perspective [49]. They reported total lifetime cost per patient at $\$ 302,100$ with 3 years versus $\$ 217,800$ for 1 year of imatinib therapy. They also found that patients on 3 years of imatinib had higher quality-adjusted life years (QALYs). Thus, incremental cost effectiveness ratio of 3 years versus 1 year of imatinib therapy was $\$ 62,600 / \mathrm{QALY}$. At a threshold of $\$ 100,000 /$ QALY, 3 year imatinib therapy was costeffective. At the same meeting Deflin and colleagues reported on the comparison of the clinical benefit of an adjuvant therapy in GIST with other adjuvant cancer therapies [50]. They showed that imatinib has one of the lowest number needed to treat amongst other adjuvant treatments, at 1 and 3 year of follow-up. Thus, both clinical and economic results now suggest treating surgically resected GIST patients with 3 years of imatinib would result in improved quality-adjusted and OS.

The Intergroup EORTC 62024 trial with randomization between two years of imatinib and observation alone has been completed and is awaiting data maturation. OS is the primary end point. A single-arm phase II five-year adjuvant imatinib trial, PERSIST5, has also completed accrual; data probably will not be available for several years.

Additionally, it is also important to assess whether certain GIST genotypic subsets benefit more-or fail to benefit at all-from adjuvant therapy with TKIs. The ACOSOG Z9001 randomized trial has confirmed certain differences between the behaviors of genetically different forms of GIST. The KIT exon 11 deletion confers a much higher risk of relapse compared with other mutational subtypes in the placebo arm, but these exon 11 mutants benefit from adjuvant imatinib to ablate this added risk [38]. The PDGFRA-driven GISTs are often more aggressive in the metastatic setting, however, they are remarkably indolent with a low risk for relapse following resection of limited-stage primary disease, similar to the wild type GIST. Given the potential toxicities and costs of imatinib, as well as the tremendous success of imatinib for recurrent disease, it is very important that adjuvant studies of imatinib be completed and analyzed in the context of molecular subtyping.

\section{Neoadjuvant therapy}

There are no published randomized trials addressing the benefit of neoadjuvant imatinib in the management of GIST. Since 2003, several case reports and small retrospective series have been published. These include a mix of patients with borderline resectable and unresectable primary disease, as well as metastatic and locally recurrent disease that is potentially amenable to gross resection [51-57].

The multicenter RTOG (Radiation Therapy Oncology Group) 0132/ACRIN (American College of Radiology Imaging Network) 6665 trial assessed neoadjuvant imatinib either in primary resectable GIST or as a planned preoperative cytoreduction agent for metastatic GIST [58]. Patients with primary GIST ( $\geq 5 \mathrm{~cm}$, Group A) or operable metastatic/recurrent GIST ( $\geq 2 \mathrm{~cm}$, Group B) were treated with neoadjuvant imatinib $(600 \mathrm{mg} /$ day) for approximately two months and maintenance imatinib after surgery for 2 years. The clinical outcomes including progression-free survival (PFS), disease-specific survival (DSS), and overall survival (OS) at a median follow-up of 5.1 years were presented at ASCO 2011 meeting [59]. The authors correlated these endpoints with duration of imatinib therapy. Sixty-three patients were originally entered (53 analyzable). There were 31 patients in Group A and 22 in Group B. Estimated 5-year PFS and DSS were 57\% A, 30\% B; and $77 \%$ A, 77\% B, respectively. Estimated 5-year OS 
was $77 \%$ for $\mathrm{A}$, and $68 \%$ for $\mathrm{B}$. Median time to progression had not been reached for Group A and was 4.4 years for Group B. In Group A, 7/11 patients progressed $>2$ years from registration; 6/7 progressing patients had stopped imatinib prior to progression. In Group B, 10/13 patients progressed $>2$ years from registration; $6 / 10$ progressing patients had stopped imatinib prior to progression. This long-term analysis suggested a high percentage of patients progressed after discontinuation of 2-year maintenance imatinib therapy following surgical resection. This trial confirmed the safety of neoadjuvant imatinib. The available data from patients treated for advanced disease suggest that maximal radiographic response to imatinib generally occurs within three to nine months.

Data from retrospective series also support the benefit of initial imatinib therapy [52-56,60]. The largest experience consisted of 46 patients who underwent surgery after imatinib. Eleven patients had a locally advanced primary tumor, while 35 had recurrent or metastatic disease [52]. All 11 patients who were treated for a locally advanced primary tumor (median 12 months of imatinib prior to resection had one complete and eight partial responses as assessed by CT) successfully underwent complete surgical resection. There was one complete pathologic response. At a median follow-up of 19.7 months post-resection, all 11 were alive and ten of them were recurrence-free. There were 11 partial responses among the 35 patients treated for recurrent/metastatic disease. Eleven patients were able to undergo a compete resection including two with a complete pathologic response. Patients with an objective partial response to imatinib (by $\mathrm{CT}$ ) were significantly more likely to undergo a complete resection. All eleven of the completely resected patients were alive at a median follow-up of 30.7 months, but six recurred at a median of 15 months, despite continuation of imatinib therapy.

A small trial suggested response rates of up to $70 \%$ after very brief periods of neoadjuvant imatinib (three to seven days), as assessed by FDG-PET and dynamic CT [61]. However, in this small prospective randomized phase II trial, there was no evidence of histologic cytoreduction (and therefore, no potential benefit in terms of reduced tumor bulk) from $\leq 7$ days of neoadjuvant imatinib, and no suggestion that intra-operative blood loss was reduced, even though blood flow to the tumor was reduced as measured by dynamic CT. Thus, the clinical benefit of very short periods of neoadjuvant imatinib (termed "nanoneoadjuvant therapy") [62] is unproven. It did prove that radiographic responses and tumor cell apoptosis occur within the first week of imatinib therapy.

Guidelines from the NCCN recommend initial treatment with imatinib for patients with marginally resectable tumors and for those who have potentially resectable disease but with the risk of significant morbidity. A daily dose of $400 \mathrm{mg}$ per day is the usual approach, although if a KIT exon 9 mutation is identified, dose escalation to $800 \mathrm{mg}$ per day is reasonable. This is also recommended in ESMO guidelines [63].

\section{Management of metastatic, unresectable or recurrent GIST}

Various mechanisms are responsible for the resistance of GISTs to conventional cytotoxic chemotherapy. In one study evaluating the differences in outcome between GIST and leiomyosarcomas, significantly higher levels of expression of P-glycoprotein $(38.4 \%$ vs. $13.4 \%)$ and MRP1 (35.4\% vs. 13.3\%) were demonstrated in the GIST cells [64].

During the 1990's, screening studies [65-67] demonstrated that a signal transduction inhibitor 571 (STI 571, imatinib mesylate) inhibits the tyrosine kinase activity of BCR-ABL as well as KIT. A multicenter US-Finland collaborative study enrolled 147 patients with metastatic GIST between July 2000 and April 2001 [68]. Radiographic responses were seen within six months in 54\% patients. These results along with the outcomes of the European Organization for Research and Treatment of Cancer (EORTC) trial 75 [69] confirmed the unparalleled activity of imatinib in controlling metastatic GIST. Despite these excellent results complete responses are rare (less than 10\%), and most patients who initially respond ultimately acquire resistance via additional mutations in KIT. The median time to progression is roughly two to three years [68,70-72], although it is longer in other series [73].

Correlative science studies have reported that the type of mutation in KIT and PDGFRA correlates with clinical response [74-77]. High dose imatinib may preferentially benefit patients with exon 9 mutations [77,78]. However, no differences in OS between low-dose and high-dose imatinib in patients with exon 9 mutations was seen [79].

Rapid disease progression was seen within months after the imatinib is stopped [80,81]. At ASCO 2011, BFR 14 trial reported the effect of interruption of imatinib therapy in patients with GIST [82]. At the same meeting, Domont et al. reported the influence of imatinib interruption and reintroduction on tumor burden in patients with GIST on the BFR 14 trial. Among randomized patients with imatinib interruption $49 \%$ experienced progressive disease of the known tumor while $51 \%$ had new lesions with concomitant progression of known lesions [83]. Thus, continuous therapy until disease progression (or lifelong if disease does not progress) is currently standard of care.

Imatinib can rapidly and dramatically decrease tumor avidity for ${ }^{18}$ FDG [84-86]. With PET imaging, the downmodulation of tumor avidity for FDG is far earlier than changes noticeable on CT scanning $[68,84,86]$. Thus PET scans can aid in the detection of primary and 
secondary imatinib resistance. CT criteria using either no growth in tumor size or a combination of tumor density and size criteria have shown a close correlation with the predictive value results of FDG-PET [22,87]. Imatinib therapy can also change the density of tumor masses in GIST $[22,68,88]$. This is an important early clinical marker of antitumor activity $[89,90]$.

\section{Resistance to imatinib}

Clonal evolution of resistant GIST may be detected after a durable objective response and disease control. Several mechanisms of resistance to imatinib in GIST may exist $[91,92]$. Pharmacokinetic variability may also contribute to drug resistance [93]. Limited clonal progression appears as the first sign of resistance to imatinib $[55,92,94]$. Dose escalation from imatinib $400 \mathrm{mg}$ daily may be considered for those with clear evidence of disease progression $[45,95]$. Sunitinib targets multiple tyrosine kinases, including the vascular endothelial growth factor receptors and PDGFR. An increasing number of reports indicate efficacy for the multi-targeted TKI sunitinib in imatinib-refractory or intolerant patients [9598]. Sunitinib has become the current standard of care for patients who have failed imatinib.

As with imatinib, the clinical activity of sunitinib is significantly influenced by the specific mutation type. Resistance to sunitinib shares similar pathogenetic mechanisms to those identified in imatinib failure, with acquisition of secondary mutations after an extended initial response to the drug [99].

Sorafenib and other TKIs (i.e. sorafenib, dasatinib, motesanib, nilotinib) have been studied in refractory GIST or after resistance to imatinib and/or sunitinib [1,100-105]. The efficacy of sorafenib was addressed in a multicenter phase II trial involving patients with either imatinib or imatinib and sunitinib-refractory GIST [106]. In a preliminary report presented at the 2011 ASCO GI Cancers symposium, the disease control rate (defined as the proportion of patients without progression as the best radiologic response) was $68 \%$, and median PFS was 5.2 months.

Nilotinib was studied in a randomized phase 3 clinical trial (ENEST g3) [107]. In this trial nilotinib was compared to a heterogeneous control arm in patients advanced/metastatic GIST who had failed imatinib and sunitinib. The control arm included best supportive care with physician choice to continue or stop imatinib or sunitinib. It failed to show significant benefit for nilotinib. Some of the most promising new approaches to overcome resistance to TKIs in GIST include targeting multiple levels of the signal transduction cascade by combining agents. This has been done, for example, by combining a kinase inhibitor such as imatinib with an inhibitor of the mTOR downstream signaling partner using the mTOR inhibitor everolimus [108]. Other strategies that are being explored include the inhibition of other pathways critical to the molecular processing of the mutant KIT or PDGFRA oncoproteins, such as the chaperone function of the heat shock protein-90 system. By inhibiting heat shock protein-90, preclinical and early clinical studies have already documented antineoplastic effects on kinase-inhibitor-resistant GIST both in vitro and in patients with progressive disease $[109,110]$.

\section{New prognostic features}

The survival was higher in GIST patients who had PDFGRA mutation as compared to KIT mutations. In patients with KIT mutations, point mutations and duplication in KIT axon 11 had better survival than GIST with other KIT mutations [111].

In a single center study of GIST patients who underwent curative surgery and were not treated with imatinib, the 2 year RFS was lower in patients who had both 557 and 558 codon mutations than in those with either 557 or 558 mutated $(p=0.03)$ [112].

Hypertension (HTN) is a side effect of drugs with VEGF signaling pathway inhibition. The relationship between sunitinib- associated HTN and treatment efficacy was analyzed. The results significantly favored the patients who developed HTN while on treatment with Sunitinib. These patients had significantly prolonged OS, PFS and TTP as compared to patients who did not develop HTN. Development of HTN during sunitinib therapy may therefore be used as a biomarker for anti-tumor efficacy [113].

At ASCO GI 2012, a mitotic index of 5/50 HPF was reported to be equivalent to a standardized uptake value (SUV) of 4.3, and a Ki67 labeling index of 5 was equivalent to SUV of 6.3 on a PET-CT. Thus, an SUV of 5 can predict the malignant potential between the high and low/intermediate risk [114].

D-dimer may be another potential marker in GIST [115]. Radiological progression (rPD) was associated with higher d-dimer levels. D-dimer levels $<1000$ had a negative predictive value for rPD of $85 \%$. Thus, d-dimer test may reduce the burden of $\mathrm{CT}$ scanning in a useful percentage of patients but will require further validation.

\section{Recent trends}

Billimoria et al. reported the evolution of multimodality management of GIST with adjuvant and neoadjuvant therapy. They found that between 2001 and 2007 use of adjuvant therapy with imatinib increased from $27 \%$ to $47 \%$. Use of neo-adjuvant therapy increased from $0 \%$ to $15 \%$ in patients with tumors $>6 \mathrm{~cm}$ [116]. Although the incidence of GIST increased from 1998 to 2001, it remained stable from 2001 to 2007. This period also saw a significant decrease in patients referred for surgery. Survival of patients 
diagnosed in 2005-2007 group improved over those diagnosed from 2002-2004 for both resected and unresected tumor, with resection of the tumor being an independent predictor of survival [117].

Italiano and co-workers reported the pattern of care, prognosis and survival in patients treated with first line Imatinib or second line Sunitinib in patients with GIST over 9 centers, including 176 patients. In the preliminary results they found that patients having secondary mutations and low serum albumin levels had the worst outcome [118].

\section{Updates from the BFR 14 trial}

BFR 14 is a prospective multicenter study from 2002 to 2009 which enrolled 434 patients. Blesius et al. reviewed 236 patients who were started on imatinib $400 \mathrm{mg}$ daily and had been on it for 5 years. Patients who did not show any progression were retrospectively analyzed. They found that patients with small tumor volume at inclusion, good performance status, having exon 11 mutation in vicinity of codon $557-558$ have higher sensitivity to imatinib and have a prolonged outcome as compared to other patients [119]. Bertucci and associates investigated factors predicting long term prognosis in patients with advanced GIST on the BFR 14 trial. The study found that female sex, performance status of 0 , platelet count $<400,000 / \mathrm{dl}$, lymphocyte count $>1500 / \mathrm{mm}^{3}$ were independent predictors of overall survival. Patients with CD 34 positivity on tumors have a better PFS [120].

\section{Conclusions and future directions}

With the molecular signature of CD117/KIT mutation, GIST has provided a great model for targeted therapy. Novel targeted agents are being explored [1]. Combination therapy of TKI inhibitors either concurrently or sequentially with agents of different classes may have synergistic effects. It is therefore predictable that further clinical research by combining agents with novel mechanisms of action for this challenging malignancy will be forthcoming [121-124].

\section{Competing interests}

The authors have no conflicts of interests.

\section{Acknowledgements}

This study was partly supported by the NYMC Blood Diseases fund (DL).

\begin{abstract}
Authors' contributions
$S A, R G, B L$ contributed to data preparation. $G L$ and $D L$ were involved in concept design, data collection, and manuscript preparation. All authors reviewed and assisted in revising the manuscript. All authors read and approved the final manuscript.
\end{abstract}

Received: 25 May 2012 Accepted: 18 June 2012

Published: 18 June 2012

\section{References}

1. Lamba G, et al: Recent advances and novel agents for gastrointestinal stromal tumor (GIST). J. Hematol \& Oncol 2012, 5:21.
2. Rubin BP, Fletcher JA, Fletcher CD: Molecular Insights into the Histogenesis and Pathogenesis of Gastrointestinal Stromal Tumors. Int J Surg Pathol 2000, 8(1):5-10.

3. Cassier PA, et al: A prospective epidemiological study of new incident GISTs during two consecutive years in Rhone Alpes region: incidence and molecular distribution of GIST in a European region. $\mathrm{Br} J \mathrm{C}$ Cancer 2010, 103(2):165-170.

4. Tran T, Davila JA, El-Serag HB: The epidemiology of malignant gastrointestinal stromal tumors: an analysis of 1,458 cases from 1992 to 2000. Am J Gastroenterol 2005, 100(1):162-168.

5. Fletcher $C D$, et al: Diagnosis of gastrointestinal stromal tumors: $A$ consensus approach. Hum Pathol 2002, 33(5):459-465.

6. Miettinen $M$, et al: Evaluation of malignancy and prognosis of gastrointestinal stromal tumors: a review. Hum Pathol 2002, 33(5):478-483.

7. Nilsson B, et al: Gastrointestinal stromal tumors: the incidence, prevalence, clinical course, and prognostication in the preimatinib mesylate era-a population-based study in western Sweden. Cancer 2005, 103(4):821-829.

8. Tryggvason G, et al: Gastrointestinal stromal tumors in Iceland, 1990-2003: the icelandic GIST study, a population-based incidence and pathologic risk stratification study. Int J Cancer 2005, 117(2):289-293.

9. Goettsch WG, et al: Incidence of gastrointestinal stromal tumours is underestimated: results of a nation-wide study. Eur J Cancer 2005, 41(18):2868-2872.

10. Tzen $C Y$, et al: Incidence of gastrointestinal stromal tumor: a retrospective study based on immunohistochemical and mutational analyses. Dig Dis Sci 2007, 52(3):792-797.

11. Kawanowa $\mathrm{K}$, et al: High incidence of microscopic gastrointestinal stromal tumors in the stomach. Hum Pathol 2006, 37(12):1527-1535.

12. Agaimy $A$, et al: Minute gastric sclerosing stromal tumors (GIST tumorlets) are common in adults and frequently show c-KIT mutations. Am J Surg Pathol 2007, 31(1):113-120.

13. Chou FF, Eng HL, Sheen-Chen SM: Smooth muscle tumors of the gastrointestinal tract: analysis of prognostic factors. Surgery 1996, 119(2):171-177.

14. Pink D, et al: Severe hypoglycemia caused by paraneoplastic production of IGF-II in patients with advanced gastrointestinal stromal tumors: a report of two cases. J Clin Oncol 2005, 23(27):6809-6811.

15. DeMatteo RP: The GIST of targeted cancer therapy: a tumor (gastrointestinal stromal tumor), a mutated gene (c-kit), and a molecular inhibitor (STI571). Ann Surg Oncol 2002, 9(9):831-839.

16. Scarpa M, et al: A systematic review on the clinical diagnosis of gastrointestinal stromal tumors. J Surg Oncol 2008, 98(5):384-392.

17. Chun HJ, et al: Gastrointestinal leiomyoma and leiomyosarcoma: CT differentiation. J Comput Assist Tomogr 1998, 22(1):69-74.

18. Levy AD, et al: Gastrointestinal stromal tumors: radiologic features with pathologic correlation. Radiographics 2003, 23(2):283-304. 456; quiz 532.

19. Ghanem N, et al: Computed tomography in gastrointestinal stromal tumors. Eur Radiol 2003, 13(7):1669-1678.

20. Burkill GJ, et al: Malignant gastrointestinal stromal tumor: distribution, imaging features, and pattern of metastatic spread. Radiology 2003, 226(2):527-532.

21. Hatch GF 3rd, et al: Tumors of the esophagus. World J Surg 2000, 24(4):401-411

22. Choi $\mathrm{H}$, et al: Correlation of computed tomography and positron emission tomography in patients with metastatic gastrointestinal stromal tumor treated at a single institution with imatinib mesylate: proposal of new computed tomography response criteria. J Clin Oncol 2007, 25(13):1753-1759.

23. Demetri GD, et al: NCCN Task Force report: update on the management of patients with gastrointestinal stromal tumors. J Natl Compr Canc Netw 2010, 8(Suppl 2):S1-S41. quiz S42-4.

24. Gayed I, et al: The role of 18 F-FDG PET in staging and early prediction of response to therapy of recurrent gastrointestinal stromal tumors. J NuCl Med 2004, 45(1):17-21

25. Kamiyama $Y$, et al: 18 F-fluorodeoxyglucose positron emission tomography: useful technique for predicting malignant potential of gastrointestinal stromal tumors. World J Surg 2005, 29(11):1429-1435.

26. Demetri GD, et al: NCCN Task Force report: management of patients with gastrointestinal stromal tumor (GIST)-update of the NCCN clinical practice guidelines. J Natl Compr Canc Netw 2007, 5(Suppl 2):S1-S29. quiz S30. 
27. Pidhorecky l, et al: Gastrointestinal stromal tumors: current diagnosis, biologic behavior, and management. Ann Surg Oncol 2000, 7(9):705-712.

28. Tio TL, Tytgat GN, den Hartog FC: Jager, Endoscopic ultrasonography for the evaluation of smooth muscle tumors in the upper gastrointestinal tract: an experience with 42 cases. Gastrointest Endosc 1990, 36(4):342-350.

29. Avritscher R, Gupta S: Gastrointestinal stromal tumor: role of interventional radiology in diagnosis and treatment. Hematol Oncol Clin North Am 2009, 23(1):129-137. ix

30. van Roggen JF Graadt, van Velthuysen ML, Hogendoorn PC: The histopathological differential diagnosis of gastrointestinal stromal tumours. J Clin Pathol 2001, 54(2):96-102.

31. Kindblom LG, et al: Gastrointestinal pacemaker cell tumor (GIPACT): gastrointestinal stromal tumors show phenotypic characteristics of the interstitial cells of Cajal. Am J Pathol 1998, 152(5):1259-1269.

32. Hirota S, et al: Gain-of-function mutations of c-kit in human gastrointestinal stromal tumors. Science 1998, 279(5350):577-580,

33. Heinrich $M C$, et al: PDGFRA activating mutations in gastrointestinal stromal tumors. Science 2003, 299(5607):708-710.

34. Miettinen M, Wang ZF, Lasota J: DOG1 antibody in the differential diagnosis of gastrointestinal stromal tumors: a study of 1840 cases. Am J Surg Pathol 2009, 33(9):1401-1408.

35. West RB, et al: The novel marker, DOG1, is expressed ubiquitously in gastrointestinal stromal tumors irrespective of KIT or PDGFRA mutation status. Am J Pathol 2004, 165(1):107-113.

36. Miettinen M, Lasota J: Gastrointestinal stromal tumors: pathology and prognosis at different sites. Semin Diagn Pathol 2006, 23(2):70-83.

37. Huang HY, et al: A modification of NIH consensus criteria to better distinguish the highly lethal subset of primary localized gastrointestinal stromal tumors: a subdivision of the original high-risk group on the basis of outcome. Surgery 2007, 141(6):748-756.

38. Corless $\mathrm{CL}$, et al: Relation of tumor pathologic and molecular features to outcome after surgical resection of localized primary gastrointestinal stromal tumor (GIST): Results of the intergroup phase III trial ACOSOG Z9001. ASCO Meeting Abstracts 2010, 28(15_suppl):10006.

39. Singer $S$, et al: Prognostic value of KIT mutation type, mitotic activity, and histologic subtype in gastrointestinal stromal tumors. J Clin Oncol 2002, 20(18):3898-3905.

40. Reith JD, et al: Extragastrointestinal (soft tissue) stromal tumors: an analysis of 48 cases with emphasis on histologic predictors of outcome. Mod Pathol 2000, 13(5):577-585.

41. Martin J, et al: Deletions affecting codons 557-558 of the c-KIT gene indicate a poor prognosis in patients with completely resected gastrointestinal stromal tumors: a study by the Spanish Group for Sarcoma Research (GEIS). J Clin Oncol 2005, 23(25):6190-6198.

42. DeVita VT, Lawrence TS, Rosenberg SA: DeVita, Hellman, and Rosenberg's cancer: principles \& practice of oncology. 9th edition. Philadelphia: Wolters Kluwer Health/Lippincott Williams \& Wilkins; 2011:2638. xlvii.

43. Dematteo RP, et al: Adjuvant imatinib mesylate after resection of localised, primary gastrointestinal stromal tumour: a randomised, double-blind, placebo-controlled trial. Lancet 2009, 373(9669):1097-1104.

44. Blackstein ME, et al: Risk assessment for tumor recurrence after surgical resection of localized primary gastrointestinal stromal tumor (GIST): North American Intergroup phase III trial ACOSOG Z9001. ASCO GI Cancer Symposium 2010, 28(suppl 4):6.

45. Blanke $C D$, et al: Long-term results from a randomized phase II trial of standard- versus higher-dose imatinib mesylate for patients with unresectable or metastatic gastrointestinal stromal tumors expressing KIT. J Clin Oncol 2008, 26(4):620-625.

46. Dematteo RP, et al: Tumor mitotic rate, size, and location independently predict recurrence after resection of primary gastrointestinal stromal tumor (GIST). Cancer 2008, 112(3):608-615.

47. Kang B, et al: A phase II study of imatinib mesylate as adjuvant treatment for curatively resected high-risk localized gastrointestinal stromal tumors. ASCO Meeting Abstracts 2009, 27(15S):e21515.

48. Joensuu $H$, et al: One vs three years of adjuvant imatinib for operable gastrointestinal stromal tumor: a randomized trial. JAMA 2012, 307(12):1265-1272.

49. Sanon $M$, et al: Cost-effectiveness of three years of adjuvant imatinib in gastrointestinal stromal tumors (GIST). ASCO Meeting Abstracts 2012, 30(4_suppl):137.
50. Deflin $\mathrm{M}$, et al: Comparison of the clinical benefit of an adjuvant therapy in gastrointestinal stromal tumors (GIST) with other adjuvant cancer therapies. ASCO Meeting Abstracts 2012, 30(4_suppl):129.

51. Lang I, et al: Case 3. Resection of originally inoperable liver metastases of gastrointestinal stromal tumor after imatinib mesylate therapy. J Clin Oncol 2003, 21(18):3538-3540.

52. Andtbacka $\mathrm{RH}$, et al: Surgical resection of gastrointestinal stromal tumors after treatment with imatinib. Ann Surg Oncol 2007, 14(1):14-24

53. Katz $D$, et al: Neoadjuvant imatinib for unresectable gastrointestinal stromal tumor. Anticancer Drugs 2004, 15(6):599-602.

54. Bonvalot $S$, et al: Impact of surgery on advanced gastrointestinal stromal tumors (GIST) in the imatinib era. Ann Surg Oncol 2006, 13(12):1596-1603.

55. Raut $C P$, et al: Surgical management of advanced gastrointestinal stromal tumors after treatment with targeted systemic therapy using kinase inhibitors. J Clin Oncol 2006, 24(15):2325-2331.

56. Scaife $C L$, et al: Is there a role for surgery in patients with "unresectable" CKIT + gastrointestinal stromal tumors treated with imatinib mesylate? Am J Surg 2003, 186(6):665-669.

57. Hohenberger $P$, et al: Indication and results of surgery following imatinib treatment of locally advanced or metastatic GI stromal tumors (GIST). ASCO Meeting Abstracts 2006, 24(18_suppl):9500.

58. Eisenberg BL, et al: Phase II trial of neoadjuvant/adjuvant imatinib mesylate (IM) for advanced primary and metastatic/recurrent operable gastrointestinal stromal tumor (GIST): early results of RTOG 0132/ACRIN 6665. J Surg Oncol 2009, 99(1):42-47.

59. Wang $D$, et al: Phase II trial of neoadjuvant/adjuvant imatinib mesylate for advanced primary and metastatic/recurrent operable gastrointestinal stromal tumors: long-term follow-up results of Radiation Therapy Oncology Group 0132. Ann Surg Oncol 2012, 19(4):1074-1080.

60. Machlenkin S, et al: The effect of neoadjuvant Imatinib therapy on outcome and survival after rectal gastrointestinal stromal tumour. Colorectal Dis 2011, 13(10):1110-1115.

61. McAuliffe JC, et al: A randomized, phase II study of preoperative plus postoperative imatinib in GIST: evidence of rapid radiographic response and temporal induction of tumor cell apoptosis. Ann Surg Oncol 2009, 16(4):910-919.

62. DeMatteo RP: Nanoneoadjuvant therapy of gastrointestinal stromal tumor (GIST). Ann Surg Oncol 2009, 16(4):799-800.

63. Casali $P G$, et al: Gastrointestinal stromal tumours: ESMO clinical recommendations for diagnosis, treatment and follow-up. Ann Oncol 2009, 20(Suppl 4):64-67.

64. Plaat $\mathrm{BE}$, et al: Soft tissue leiomyosarcomas and malignant gastrointestinal stromal tumors: differences in clinical outcome and expression of multidrug resistance proteins. J Clin Oncol 2000, 18(18):3211-3220.

65. Druker BJ, et al: Effects of a selective inhibitor of the Abl tyrosine kinase on the growth of Bcr-Abl positive cells. Nat Med 1996, 2(5):561-566.

66. Buchdunger $E$, et al: Inhibition of the Abl protein-tyrosine kinase in vitro and in vivo by a 2-phenylaminopyrimidine derivative. Cancer Res 1996, 56(1):100-104.

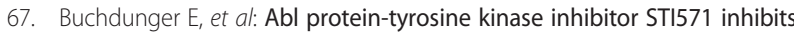
in vitro signal transduction mediated by c-kit and platelet-derived growth factor receptors. J Pharmacol Exp Ther 2000, 295(1):139-145.

68. Demetri GD, et al: Efficacy and safety of imatinib mesylate in advanced gastrointestinal stromal tumors. N Engl J Med 2002, 347(7):472-480

69. van Oosterom AT, et al: Safety and efficacy of imatinib (STI571) in metastatic gastrointestinal stromal tumours: a phase I study. Lancet 2001, 358(9291):1421-1423.

70. Verweij J, et al: Progression-free survival in gastrointestinal stromal tumours with high-dose imatinib: randomised trial. Lancet 2004, 364(9440):1127-1134.

71. Van Glabbeke $M$, et al: Initial and late resistance to imatinib in advanced gastrointestinal stromal tumors are predicted by different prognostic factors: a European Organisation for Research and Treatment of Cancer-Italian Sarcoma Group-Australasian Gastrointestinal Trials Group study. J Clin Oncol 2005, 23(24):5795-5804.

72. Rutkowski $P$, et al: Predictive factors for long-term effects of imatinib therapy in patients with inoperable/metastatic CD117(+) gastrointestinal stromal tumors (GISTs). J Cancer Res Clin Oncol 2007, 133(9):589-597.

73. Armbrust T, et al: Does imatinib turn recurrent and/or metastasized gastrointestinal stromal tumors into a chronic disease? - single center experience. Eur J Gastroenterol Hepatol 2009, 21(7):819-823. 
74. Heinrich MC, et al: Kinase mutations and imatinib response in patients with metastatic gastrointestinal stromal tumor. J Clin Oncol 2003, 21(23):4342-4349.

75. Debiec-Rychter M, et al: Use of c-KIT/PDGFRA mutational analysis to predict the clinical response to imatinib in patients with advanced gastrointestinal stromal tumours entered on phase I and II studies of the EORTC Soft Tissue and Bone Sarcoma Group. Eur J Cancer 2004, 40(5):689-695.

76. Corless $\mathrm{CL}$, et al: PDGFRA mutations in gastrointestinal stromal tumors: frequency, spectrum and in vitro sensitivity to imatinib. J Clin Oncol 2005, 23(23):5357-5364

77. Heinrich MC, et al: Correlation of kinase genotype and clinical outcome in the North American Intergroup Phase III Trial of imatinib mesylate for treatment of advanced gastrointestinal stromal tumor: CALGB 150105 Study by Cancer and Leukemia Group B and Southwest Oncology Group. J Clin Oncol 2008, 26(33):5360-5367.

78. Debiec-Rychter M, et al: KIT mutations and dose selection for imatinib in patients with advanced gastrointestinal stromal tumours. Eur J Cancer 2006, 42(8):1093-1103.

79. Van Glabbeke MM, et al: Comparison of two doses of imatinib for the treatment of unresectable or metastatic gastrointestinal stromal tumors (GIST): A meta-analyis based on 1,640 patients (pts). ASCO Meeting Abstracts 2007, 25(18 suppl):10004.

80. Blay JY, et al: Prospective multicentric randomized phase III study of imatinib in patients with advanced gastrointestinal stromal tumors comparing interruption versus continuation of treatment beyond 1 year: the French Sarcoma Group. J Clin Oncol 2007, 25(9):1107-1113.

81. Le Cesne $A$, et al: Discontinuation of imatinib in patients with advanced gastrointestinal stromal tumours after 3 years of treatment: an open-label multicentre randomised phase 3 trial. Lancet Oncol 2010, 11(10):942-949.

82. Blay JY, Perol D, Le Cesne A: Imatinib rechallenge in patients with advanced gastrointestinal stromal tumors. Ann Oncol 2012, 23. doi:10.1093/annonc/mdr622.

83. Domont J, et al: Influence of imatinib interruption and imatinib rechallenge on the residual tumor volume in patients with advanced GIST: Results of the BFR14 prospective French Sarcoma Group randomized phase III trial. ASCO Meeting Abstracts 2011, 29(15_suppl):10054.

84. Stroobants $\mathrm{S}$, et al: 18FDG-Positron emission tomography for the early prediction of response in advanced soft tissue sarcoma treated with imatinib mesylate (Glivec). Eur J Cancer 2003, 39(14):2012-2020.

85. Antoch $\mathrm{G}$, et al: Comparison of PET, CT, and dual-modality PET/CT imaging for monitoring of imatinib (STI571) therapy in patients with gastrointestinal stromal tumors. J Nucl Med 2004, 45(3):357-365.

86. Van den Abbeele AD, Badawi RD: Use of positron emission tomography in oncology and its potential role to assess response to imatinib mesylate therapy in gastrointestinal stromal tumors (GISTs). Eur J Cancer 2002, 38(Suppl 5):S60-S65.

87. Benjamin RS, et al: We should desist using RECIST, at least in GIST. J Clin Oncol 2007, 25(13):1760-1764.

88. Joensuu $H$, et al: Effect of the tyrosine kinase inhibitor STI571 in a patient with a metastatic gastrointestinal stromal tumor. N Engl J Med 2001, 344(14):1052-1056

89. Bechtold RE, et al: Cystic changes in hepatic and peritoneal metastases from gastrointestinal stromal tumors treated with Gleevec. Abdom Imaging 2003, 28(6):808-814

90. Linton KM, Taylor MB, Radford JA: Response evaluation in gastrointestinal stromal tumours treated with imatinib: misdiagnosis of disease progression on $\mathrm{CT}$ due to cystic change in liver metastases. $\mathrm{Br} J$ Radio 2006, 79(944):e40-e44.

91. Heinrich $M C$, et al: Molecular correlates of imatinib resistance in gastrointestinal stromal tumors. J Clin Oncol 2006, 24(29):4764-4774.

92. Desai J, et al: Clonal evolution of resistance to imatinib in patients with metastatic gastrointestinal stromal tumors. Clin Cancer Res 2007, 13(18 Pt 1):5398-5405

93. Eechoute $K$, et al: Drug transporters and imatinib treatment: implications for clinical practice. Clin Cancer Res 2011, 17(3):406-415.

94. Shankar S, et al: Gastrointestinal stromal tumor: new nodule-within-a -mass pattern of recurrence after partial response to imatinib mesylate. Radiology 2005, 235(3):892-898

95. Debiec-Rychter $M$, et al: Mechanisms of resistance to imatinib mesylate in gastrointestinal stromal tumors and activity of the PKC412 inhibitor against imatinib-resistant mutants. Gastroenterology 2005, 128(2):270-279.
96. Demetri GD, et al: Molecular target modulation, imaging, and clinical evaluation of gastrointestinal stromal tumor patients treated with sunitinib malate after imatinib failure. Clin Cancer Res 2009, 15(18):5902-5909.

97. Heinrich MC, et al: Primary and secondary kinase genotypes correlate with the biological and clinical activity of sunitinib in imatinib-resistant gastrointestinal stromal tumor. J Clin Oncol 2008, 26(33):5352-5359.

98. Demetri GD, et al: Efficacy and safety of sunitinib in patients with advanced gastrointestinal stromal tumour after failure of imatinib: a randomised controlled trial. Lancet 2006, 368(9544):1329-1338.

99. Guo $T$, et al: Mechanisms of sunitinib resistance in gastrointestinal stromal tumors harboring KITAY502-3ins mutation: an in vitro mutagenesis screen for drug resistance. Clin Cancer Res 2009, 15(22):6862-6870.

100. Joensuu $H$, et al: Phase II, open-label study of PTK787/ZK222584 for the treatment of metastatic gastrointestinal stromal tumors resistant to imatinib mesylate. Ann Oncol 2008, 19(1):173-177.

101. Montemurro $M$, et al: Nilotinib in the treatment of advanced gastrointestinal stromal tumours resistant to both imatinib and sunitinib. Eur J Cancer 2009, 45(13):2293-2297.

102. Dewaele $B$, et al: Activity of dasatinib, a dual SRC/ABL kinase inhibitor, and IPI-504, a heat shock protein 90 inhibitor, against gastrointestinal stromal tumor-associated PDGFRAD842V mutation. Clin Cancer Res 2008 14(18):5749-5758

103. Demetri GD, et al: A phase I study of single-agent nilotinib or in combination with imatinib in patients with imatinib-resistant gastrointestinal stromal tumors. Clin Cancer Res 2009, 15(18):5910-5916.

104. Benjamin RS, et al: Efficacy and safety of motesanib, an oral inhibitor of VEGF, PDGF, and Kit receptors, in patients with imatinib-resistant gastrointestinal stromal tumors. Cancer Chemother Pharmacol 2011, 68(1):69-77.

105. Wiebe L, et al: Activity of sorafenib (SOR) in patients (pts) with imatinib (IM) and sunitinib (SU)-resistant (RES) gastrointestinal stromal tumors (GIST): A phase II trial of the University of Chicago Phase II Consortium. ASCO Meeting Abstracts 2008, 26(15_suppl):10502.

106. Campbell NP, et al: Final results of a University of Chicago phase II consortium trial of sorafenib (SOR) in patients (pts) with imatinib (IM)and sunitinib (SU)-resistant (RES) gastrointestinal stromal tumors (GIST). ASCO Meeting Abstracts 2011, 29(4_suppl):4.

107. Reichardt $P$, et al: Phase III study of nilotinib versus best supportive care with or without a TKI in patients with gastrointestinal stromal tumors resistant to or intolerant of imatinib and sunitinib. Ann Oncol 2012, 23 doi:10.1093/annonc/mdr598.

108. Schoffski $P$, et al: A phase I-II study of everolimus (RAD001) in combination with imatinib in patients with imatinib-resistant gastrointestinal stromal tumors. Ann Oncol 2010, 21(10):1990-1998.

109. Bauer $\mathrm{S}$, et al: Heat shock protein 90 inhibition in imatinib-resistant gastrointestinal stromal tumor. Cancer Res 2006, 66(18):9153-9161.

110. Demetri GD, et al: An open-label phase II study of the Hsp90 inhibitor ganetespib (STA-9090) in patients (pts) with metastatic and/or unresectable GIST. ASCO Meeting Abstracts 2011, 29(15_suppl):10011.

111. Mazurenko NN, et al: Prognostic relevance of genetic aberrations in gastrointestinal stromal tumors. ASCO GI Cancery Symposium 2011, 29(Suppl 4):49.

112. Watanabe T, et al: Impact of c-kit mutations, including codons 557 and/or 558 , on the recurrence-free survival after curative surgery in patients with GIST. ASCO GI Cancer Symposium 2011, 29(Suppl 4):12.

113. George $S$, et al: Hypertension (HTN) as a potential biomarker of efficacy in patients (pts) with gastrointestinal stromal tumor (GIST) treated with sunitinib (SU). ASCO GI Cancer Symposium 2011, 29(Suppl 4):38.

114. Yoshikawa $\mathrm{K}$, et al: The utility of PET-CT in predicting malignant potential of GIST. ASCO GI Cancer Symposium 2012, 30(Suppl 4):38.

115. Seligmann JF, et al: D-dimers as a tumor marker in GIST: Can it reduce the frequency of $\mathrm{CT}$ scanning in patients receiving palliative imatinib? ASCO GI Cancer Symposium 2012, 30(Suppl 4):119.

116. Bilimoria KY, et al: Incorporation of adjuvant therapy into the multimodality management of gastrointestinal stromal tumors of the stomach in the United States. Ann Surg Oncol 2012, 19(1):184-191.

117. Witkowski ER, et al: Nationwide trends in diagnosis and outcomes of gastrointestinal stromal tumors in the era of targeted therapy. ASCO Gl Cancer Symposium 2011, 29(Suppl 4):121. 
118. Italiano A, et al: Patterns of Care, Prognosis, and Survival in Patients with Metastatic Gastrointestinal Stromal Tumors (GIST) Refractory to First-Line Imatinib and Second-Line Sunitinib. Ann Surg Oncol 2012, 19(5):1551-1559.

119. Blesius $A$, et al: Who are the long responders to imatinib (IM) in patients with advanced GIST? Results of the BFR14 prospective French Sarcoma Group randomized phase III trial. ASCO Meeting Abstracts 2011, 29(15_suppl):10048.

120. Bertucci $F$, et al: Prognostic factors for progression-free and overall survival in patients with advanced GIST treated with standard-dose imatinib (IM): Results from the BFR14 phase III trial of the French Sarcoma Group. ASCO Meeting Abstracts 2011, 29(15_suppl):e20506.

121. Yuan Y, et al: Novel targeted therapeutics: inhibitors of MDM2, ALK and PARP. J Hematol Oncol 2011, 4:16.

122. Tan J, et al: Novel histone deacetylase inhibitors in clinical trials as anti-cancer agents. J Hematol Oncol 2010, 3(1):5.

123. Fremin C, Meloche S: From basic research to clinical development of MEK1/2 inhibitors for cancer therapy. J Hematol Oncol 2010, 3(1):8.

124. Elbaz $\mathrm{H}$, et al: Digitoxin and its analogs as novel cancer therapeutics. Exp Hematol Oncol 2012, 1(1):4

doi:10.1186/2162-3619-1-14

Cite this article as: Lamba et al:: Current management and prognostic features for gastrointestinal stromal tumor (GIST). Experimental

Hematology \& Oncology 2012 1:14

\section{Submit your next manuscript to BioMed Central and take full advantage of:}

- Convenient online submission

- Thorough peer review

- No space constraints or color figure charges

- Immediate publication on acceptance

- Inclusion in PubMed, CAS, Scopus and Google Scholar

- Research which is freely available for redistribution 